\title{
PROSTATECTOMÍA RADICAL LAPAROSCÓPICA: EXPERIENCIA PRELIMINAR
}

\author{
J. ESTÉBANEZ ZARRANZ, J. AMÓN SESMERO, C. CONDE REDONDO, J. SANTOS \\ LARGO, D. ALONSO FERNÁNDEZ, A. ROBLES SAMANIEGO, \\ N. DEL VALLE GONZÁLEZ, J.Mª MARTÍNEZ-SAGARRA OCEJA
}

Servicio de Urología. Hospital del Río Hortega. Valladolid.

Actas Urol Esp. 27 (5): 370-378, 2003

\section{RESUMEN}

"PROSTATECTOMÍA RADICAL LAPAROSCÓPICA: EXPERIENCIA PRELIMINAR"

INTRODUCCIÓN: Con intención de disminuir la morbilidad de la prostatectomá radical, se ha desarrollado la técnica de la prostatectomía radical laparoscópica (PRL). Presentamos en este trabajo nuestra experiencia preliminar.

MATERIAL Y MÉTODOS: Desde el 24 de enero del 2002 hasta el 5 de mayo del 2002, hemos operado 8 pacientes. A uno realizamos linfadenectomía unilateral. Se utiliza una técnica transperitoneal con algunas variantes a la descrita en el Instituto Montsouris.

RESULTADOS: El tiempo quirúrgico medio ha sido de 356 minutos (540-240). Ningún paciente ha precisado de transfusión. Complicaciones intraoperatorias: dos lesiones vesicales y una lesión de arteria epigástrica. Complicaciones post-operatorias: un ileo paralítico, dos neuropraxias, cuatro fístulas de la anastomosis. Todas se resolvieron con medidas conservadoras y ningún paciente precisó reconversión a cirugía abierta. Ninguno presentó bordes positivos. De los pacientes controlados todos tenían PSA inferior a 0,1 al mes de la intervención.

CONCLUSIONES: La PRL es una técnica difícil pero creemos que la curva de aprendizaje no será tan larga como en las primeras series. Los resultados oncológicos son similares a la cirugía abierta, pero ofrece pequeñas ventajas en muchos aspectos (sangrado, días de sondaje, estancia hospitalaria, retorno a la actividad, continencia, función sexual, cosmética, dolor post-operatorio...).

PALABRAS CLAVE: Cáncer de próstata. Prostatectomía radical. Laparoscopia.

\section{ABSTRACT}

"LAPAROSCOPIC RADICAL PROSTATECTOMY. PRELIMINAR EXPERIENCE"

OBJETIVE: Laparoscopic technique has been developped with the aim to decrese the morbidity of the open radical prostatectomy.

MATERIAL AND METHODS: From january 2002 to may 2002, 8 patients were treated for prostate cancer with laparoscopic radical prostatectomy. Unilateral linfadenectomy has been carried out in oly one patient. We usually employ the transperitoneal technique published by the Montsouris Institute, with some modifications.

RESULTS: The main surgical time was 356 minuts (540-240). Transfusion wasn't needed in any case. Intraoperative complications were: 2 bladder injuries, 1 bleeding of the epigastric artery. Postoperative complications were: 1 ileus, 2 compressive neurapraxia, 4 anastomotic fistutas. All complications were treated conservatively. No patient were converted to open surgery. Surgical limits were negatifs in all cases, and the PSA rate was less than $0.1 \mathrm{ng} / \mathrm{ml}$ in the first month follow up.

CONCLUSION: Laparoscopic radical prostatectomy is a dificult technique, but we think that, the learning curve is getting lower and lower than in the first series. Oncologic results with this technique is similar to the open one. However, laparoscopic approach shows us some benefits such as less bleeding, less time catheterisation, less hospital stay, better continence, better sexual fonction, better stetic result, less postoperative pain, and finally an earlier back to work.

KEY WORDS: Prostate cancer. Radical prostatectomy. Laparoscopy. 
$\mathrm{L}^{\mathrm{a}}$ a prostatectomía radical es el tratamiento aconsejado en el cáncer de próstata localizado en los pacientes con buena salud y expectativa de vida de por lo menos 10 años. La operación erradica el cáncer en la mayoría de los casos, pero no está exenta de morbilidad y mortalidad $^{1}$. Con intención de disminuir la morbilidad de la cirugía convencional, se ha desarrollado la técnica de la PRL. La primera publicación de esta técnica es del Schuessler ${ }^{2}$, pero debido a sus dificultades técnicas, concluye que la PRL no es una alternativa eficaz a la prostatectomía abierta $^{3}$. Sin embargo, Guillonneau y Vallancien ${ }^{4}$ en el Instituto Montsouris de París han desarrollado esta técnica, consiguiendo que actualmente sea una operación que se realiza de rutina en muchos hospitales. El gran número de pacientes que han operado y los excelentes resultados que han publicado, nos ha animado a tratar a nuestros pacientes con esta técnica.

Presentamos en este trabajo nuestros primeros 8 casos, haciendo hincapié sobre todo en los problemas técnicos.

\section{MATERIAL Y MÉTODOS}

Desde el 24 de enero del 2002 hasta el 5 de mayo del 2002, hemos operado 8 pacientes de PRL diagnosticados de cáncer de próstata en estadio localizado. La edad media fue de 64,8 (5771). Un paciente había sido operado de apendicectomía y tres tenían una herniorrafia previa. El paciente 7 había sido tratado con bloqueo hormonal completo durante 2 meses. El PSA estaba entre 4,5 y 14,4, ya que seleccionamos pacientes con PSA menor de 15 para no tener que realizar linfadenectomía. Sólo un caso tenía PSA de 17,6 y realizamos linfadenectomía unilateral. El estadío clínico era: 7 casos T1C y 1 caso T2A. A todos los pacientes se les diagnosticó mediante biopsia transrectal guiada por ultrasonidos. El peso de las próstatas medidas en la ecografía era de 62 gr. de media (29-165). El Gleason y el número de biopsias positivas lo reflejamos en la Tabla I. Todos ellos tenian un factor de riesgo anestésico (ASA) de II.

\section{Técnica}

Cuidados preoperatorios: La profilaxis antibiótica la realizamos con una dosis de una cefalosporina de $3^{\mathrm{a}}$ generación preoperatoria. La profilaxis antitrombótica la realizamos con una heparina de bajo peso molecular previa a la intervención, que mantenemos durante 15 días. Así mismo ponemos un vendaje compresivo en extremidades inferiores. La preparación intestinal la realizamos con fosfato sódico.

Colocación del paciente: La operación se realiza bajo anestesia general. Se coloca al paciente en decúbito supino, con los brazos paralelos al cuerpo y las piernas entreabiertas y sujetadas con perneras de tal forma que la torre de laparoscopia quede entre ellas. Se sujeta al paciente a la mesa para que no se deslice cuando se coloque en posición de Trendelemburg de $45^{\circ}$. Se coloca una sonda vesical. Se introduce una aguja de Veress periumbilical y se insufla la cavidad abdominal con $\mathrm{CO} 2$ a una presión

\section{TABLA I}

CARACTERÍSTICAS DE LOS PACIENTES

\begin{tabular}{|c|c|c|c|c|c|c|}
\hline & Edad & Antecedentes & PSA & Gleason & $\begin{array}{c}\text { № biopsia+t } \\
\text { № biopsia }\end{array}$ & $\begin{array}{c}\text { Volumen } \\
\text { prostático }\end{array}$ \\
\hline 1 & 67 & Apendicectomía & 14,4 & $3+3$ & $7 / 7$ & 45 \\
\hline 2 & 69 & Herniorrafia & 5,7 & $2+2$ & $1 / 4$ & 165 \\
\hline 3 & 59 & Herniorrafia & 7,8 & $3+3$ & $2 / 8$ & 41 \\
\hline 4 & 57 & Herniorrafia & 4,5 & $3+3$ & $4 / 8$ & 29 \\
\hline 5 & 58 & & 9,4 & $2+3$ & $2 / 8$ & 41 \\
\hline 6 & 67 & & 12,7 & $2+2$ & $4 / 8$ & 55 \\
\hline 7 & 71 & Bloqueo hormonal & 17,6 & $2+2$ & $1 / 6$ & 52 \\
\hline 8 & 71 & & 11,7 & $3+2$ & $1 / 6$ & 68 \\
\hline
\end{tabular}


entre 12 y $13 \mathrm{mmHg}$. Se introduce un trocar de $10 \mathrm{~mm}$ periumbilical por donde se pasa la óptica. Bajo visión directa se introducen otros 4 trocares: uno de $5 \mathrm{~mm}$ en la fosa ilíaca izquierda, otro de $5 \mathrm{~mm}$ en la línea media entre el ombligo y el pubis, otros dos trocares (uno de 5 y otro de $10 \mathrm{~mm}$ ) en el lado derecho en la línea entre el ombligo y la cresta ilíaca. El cirujano se coloca en el lado izquierdo del paciente sobre una plataforma, y los ayudantes en el lado derecho.

Pasos quirúrgicos: En líneas generales seguimos los pasos descritos por Guillonneau y Vallancien.

1) Disección de vesículas seminales: En el fondo de saco de Douglas se aprecian claramente dos arcos (Fig. 1): uno superior que corresponde a la localización de los uréteres y otro inferior donde se localizan los deferentes. Se realiza una incisión sobre el arco inferior y se diseca cuidadosamente hasta encontrar los deferentes. Se coagulan con pinza bipolar y se seccionan. Por detrás de ellos se localiza siempre la arteria diferencial que es preciso coagular también. Se localizan después las vesículas seminales que se disecan hasta que se puedan movilizar completamente (Fig. 2).

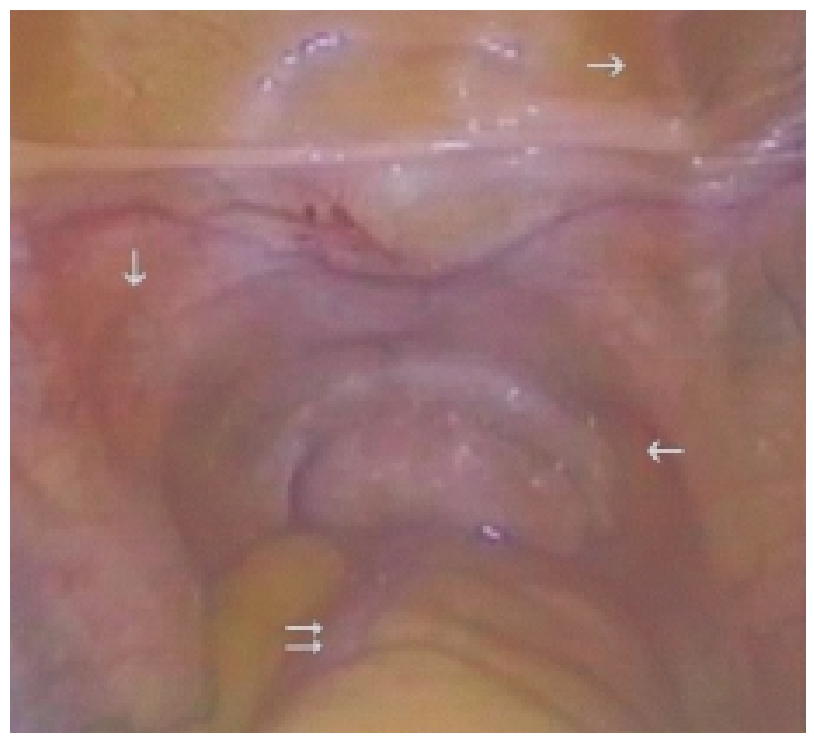

FIGURA 1. Anatomia del fondo de saco de Douglas. Se puede observar el recto $(\rightarrow)$, el ligamento umbilical $(\rightarrow)$ y dos arcos, uno superior por donde transcurren los uréteres $(\downarrow)$ y otro inferior donde están los deferentes y las vesiculas seminales $(\leftarrow)$.
2) Apertura de la fascia de Denonvilliers: Se cogen las vesículas seminales con una pinza de agarre y se tracciona de ellas hacia arriba, lo que permite visualizar claramente la fascia de Denonvilliers. Se incinde la fascia con corte frío hasta ver la grasa perirrectal (Fig. 3).

3) Disección vesical: Se llena la vejiga con $120 \mathrm{cc}$ de suero, con lo que se puede distinguir los bordes de la vejiga. Se incinde el peritoneo entre la vejiga y el ligamento umbilical en ambos lados con el límite inferior en el deferente. Se va ampliando la incisión hasta el uraco que es preciso seccionar lo más alto posible para no lesionar la vejiga (Fig. 4). Se va disecando la cara anterior de la vejiga hasta llegar al pubis.

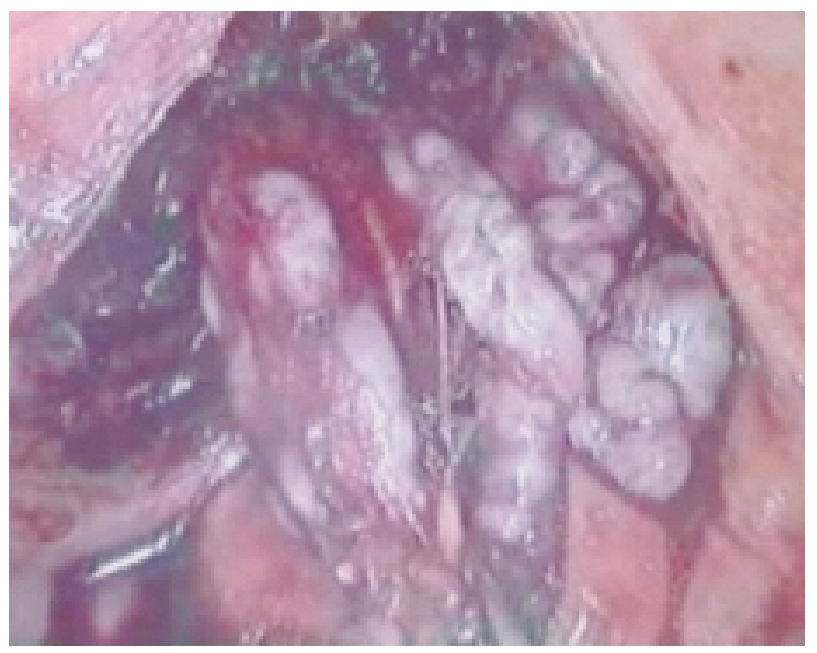

FIGURA 2. Vesiculas seminales disecadas.

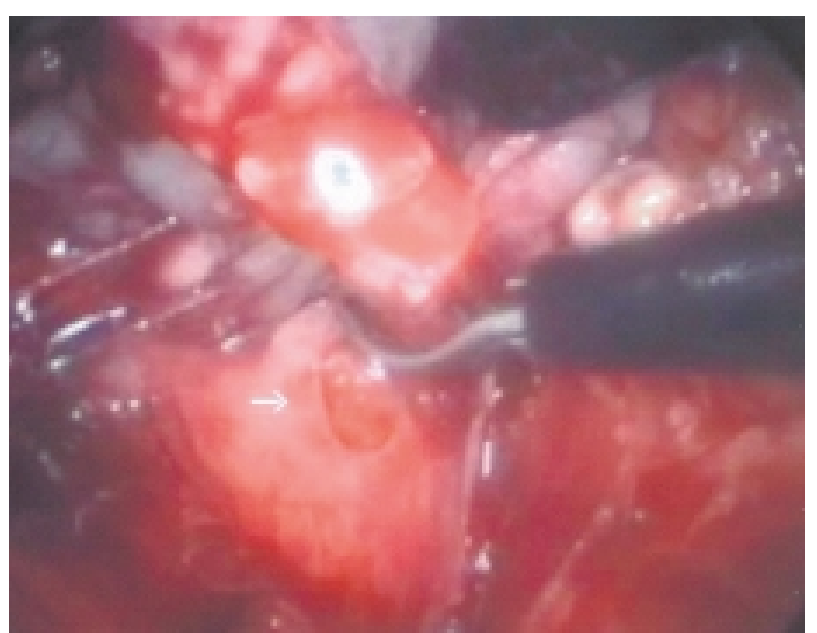

FIGURA 3. Tirando de las vesiculas hacia arriba, se observa la fascia de Denonbillers. Cuando se abre se aprecia la grasa prerectal $(\rightarrow)$. 


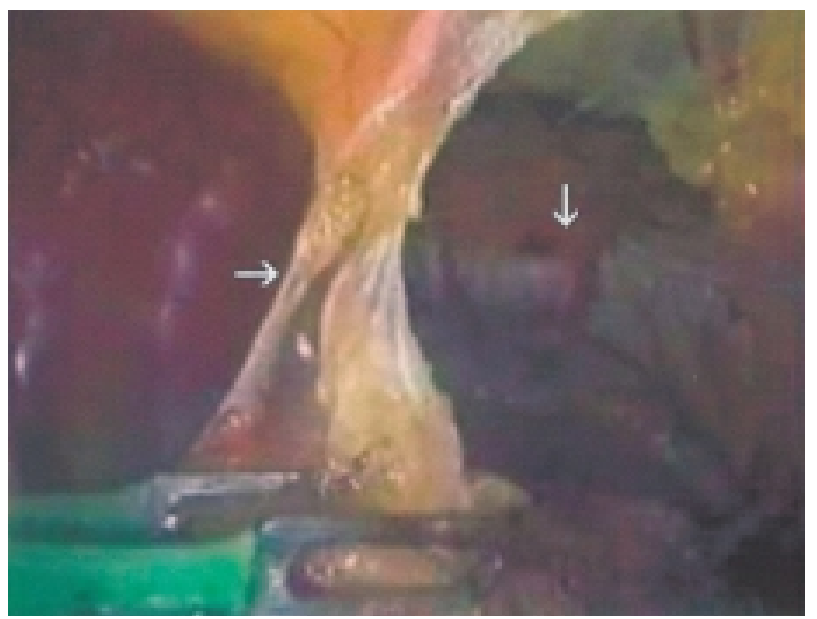

FIGURA 4. Sección del uraco $(\rightarrow)$. Por detrás se aprecia el espacio de Retzius y el pubis $(\downarrow)$.

4) Apertura de fascia endopélvica: Se libera la grasa del espacio de Retzius, hasta descubrir la fascia endopélvica. Entre los dos ligamentos puboprostáticos se distingue la vena dorsal superficial que es preciso coagular con pinzas bipolares. En ocasiones hemos utilizado el sistema de sellado de vasos LigaSure ${ }^{\mathrm{TM}}$ (Tyco Healthcare). Se incinde la fascia endopélvica (Fig. 5) y se seccionan los ligamentos puboprostáticos (Fig. 6), quedando expuesto claramente el complejo de la vena dorsal y la uretra por debajo.

5) Ligadura de la vena dorsal: La realizamos con sutura reabsorbible de 2-0, aguja de 36 y nudos intracorpóreos. En alguna ocasión hemos utilizado el dispositivo para la colocación de puntos laparoscópicos EndoStitch ${ }^{\mathrm{TM}}$ (Тyco), pero nos

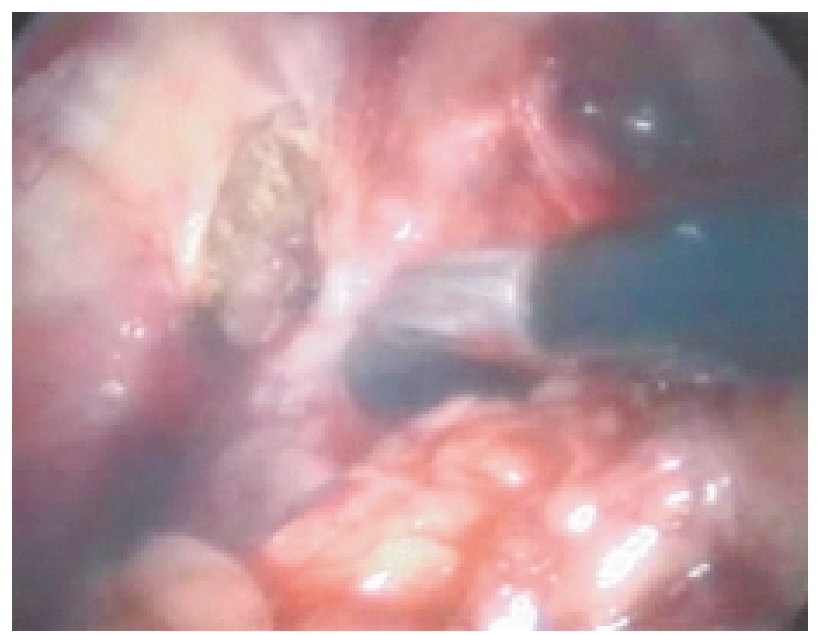

FIGURA 5. Apertura de la fascia endopélvica.

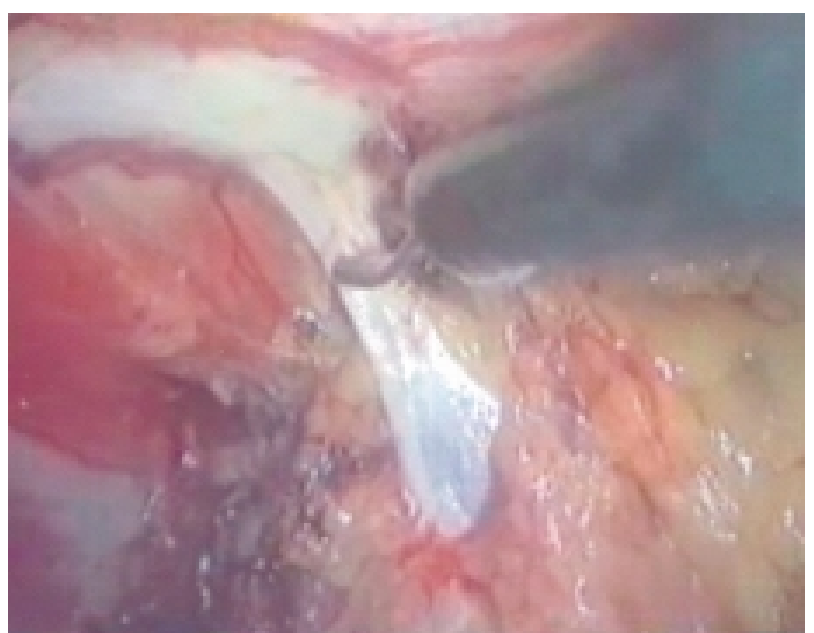

FIGURA 6. Sección de ligamento puboprostático.

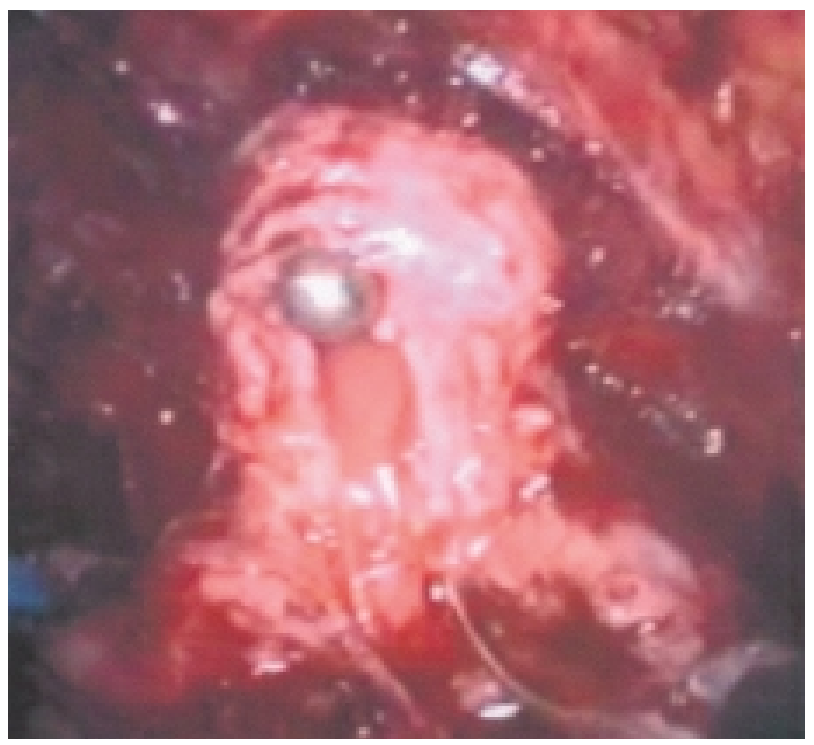

FIGURA 7. Disección de la uretra.

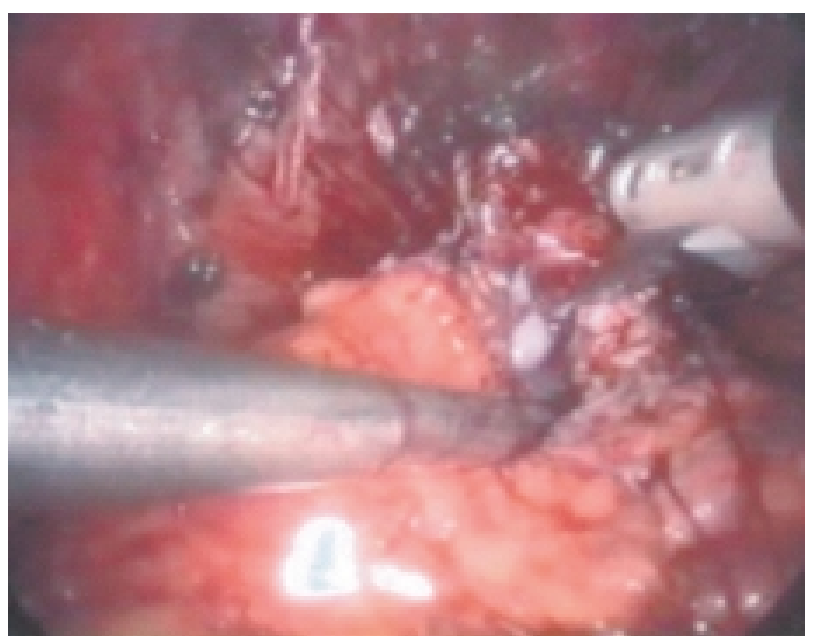

FIGURA 8. Sutura uretro-vesical con EndoStitch. Se puede observar la sonda colocada. 
ha resultado difícil de usar ya que sus ramas dificultan la visión del complejo dorsal. En el último caso hemos utilizado una aguja recta, que creemos es más útil que la curva. Asímismo realizamos un sellado de vasos con el LigaSure.

6) Disección del cuello vesical: Se realiza la incisión entre la vejiga y la próstata guiándonos en la línea donde desaparece la grasa perivesical anterior. Realizamos la coagulación con pinzas bipolares y buscamos el plano avascular de separación entre la próstata y la vejiga. Sección de la mucosa vesical a nivel del cuello ayudados de un Béniqué introducido por uretra y que luego utilizamos para levantar la próstata. De esta forma podemos incindir el labio posterior del cuello y disecar la pared posterior del cuello hasta encontrar las vesículas seminales previamente disecadas.

7) Disección lateral: Una vez completado la disección del cuello, se tira de las vesículas seminales con una pinza de agarre hacia arriba exponiéndose claramente los pedículos laterales. Se pueden coagular con pinzas bipolares. En una ocasión utilizamos EndoGia para seccionarlos, pero lo que hemos encontrado más cómodo es el LigaSure. Nunca hemos conservado las bandeletas neurovasculares.

8) Sección apical: Se secciona en primer lugar el complejo de la vena dorsal. Se expone la cara anterior de la uretra y ayudados por un Béniqué la cortamos con corte frío (Fig. 7). La sección del músculo rectouretral deja libre la próstata, que dejamos temporalmente en la fosa paracólica derecha.

9) Anastomosis uretrovesical: En los dos primeros casos realizamos la sutura tal y como la describe Guillonneau y Vallancien, es decir 10 puntos con sutura reabsorbible de 3-0 y nudos intracorpóreos. En los siguientes casos fuimos dando cada vez más puntos con el dispositivo EndoStitch $^{\mathrm{TM}}$ (Fig. 8). En los dos últimos casos toda la sutura la realizamos con este instrumento que nos ahorra mucho tiempo y con el que no perdemos seguridad en la anastomosis. Además nos permite introducir la sonda bajo visión directa cuando se finaliza la sutura de la cara posterior, sin miedo de que cuando se suture la cara anterior se pinche el globo del catéter.
10) Finalización de la operación: Finalizada la sutura, se infla el balón de la sonda, y se comprueba la estanqueidad con un lavado de la sonda. Se introduce el drenaje (un tubo de silicona multiperforado) por el trocar de 5 más externo del lado derecho. Se cambia la óptica al otro trocar de 10. Se introduce una bolsa por el trocar del ombligo que es por donde se extrae la pieza, ampliando la incisión si es preciso. Se extrae los otros trocares bajo visión directa. Si alguno sangra, se dan unos puntos con una aguja de Carter-Thomason.

\section{RESULTADOS}

Complicaciones intraoperatorias: El sangrado lo especificamos en la Tabla II. La medida la hemos realizado por lo extraído en el vaso del aspirador. Ningún paciente ha precisado de transfusión. En dos ocasiones se produjo la apertura accidental de la vejiga durante la disección de las vesículas seminales. Esta complicación fue resuelta mediante la sutura de la misma con puntos sueltos intracorpóreos. En ningún caso se observó una repercusión clínica. En otro caso se produjo una lesión de la arteria epigástrica en el momento de la introducción del trocar de $10 \mathrm{~mm}$ de la derecha. Se resolvió mediante la colocación de un punto con el dispositivo EndoClose ${ }^{\mathrm{TM}}$ (Tyco) sin observarse posteriormente repercusión clínica. Ningún caso precisó reconversión a cirugía abierta. El tiempo quirúrgico medio ha sido de 356 minutos (540-240), medidos desde la introducción de la aguja de Veress hasta el último punto de piel.

Complicaciones post-operatorias: Un paciente presentó un ileo paralítico que precisó sonda nasogástrica durante 4 días. Los dos primeros pacientes presentaron una neuropraxia en una pierna que aunque no les dificultaba la deambulación les produjo sobretodo al primer caso parestesias y dolores durante 3 meses. 4 pacientes presentaron fístula de la anastomosis (definida como la fuga de orina por más de 6 días). Todas se resolvieron manteniendo la sonda vesical por más tiempo. El tiempo medio de ingreso ha sido de 10 días (4-22), pero si se excluyen los pacientes que presentaron fistula uretro-vesical el tiempo de estancia hospitalaria se reduce a 5 días. Ocurre parecido con el tiempo de perma- 
RESULTADOS

\begin{tabular}{|c|c|c|c|c|c|c|c|}
\hline & Tiempo & Sangrado & Días de ingreso & Días con sonda & Complicaciones & Transfusión & Continencia \\
\hline 1 & 540 & 400 & 11 & 15 & $\begin{array}{l}\text { Íleo, fístula, } \\
\text { neuropraxia }\end{array}$ & No & $\mathrm{Si}$ \\
\hline 2 & 420 & 500 & 5 & 11 & & No & $\mathrm{Si}$ \\
\hline 3 & 360 & 300 & 4 & 8 & $\begin{array}{l}\text { Lesión vesical, } \\
\text { neuropraxia }\end{array}$ & No & $\mathrm{Si}$ \\
\hline 4 & 300 & 800 & 12 & 18 & $\begin{array}{l}\text { Lesión epigástrica, } \\
\text { fístula }\end{array}$ & No & $\mathrm{Si}$ \\
\hline 5 & 420 & 500 & 5 & 9 & & No & $\mathrm{Si}$ \\
\hline 6 & 240 & 300 & 6 & 10 & Lesión vesical & No & $\mathrm{Si}$ \\
\hline 7 & 270 & 300 & 20 & 19 & Fístula & No & No \\
\hline 8 & 300 & 300 & 22 & 20 & Fistula & No & No \\
\hline
\end{tabular}

nencia de la sonda vesical, que ha sido de 13,8 días de media (8-21), pero si excluimos a los pacientes fistulizados se reduce a 9,5 días. En la actualidad 6 pacientes son continentes totales (no necesitan ningún tipo pañal). A destacar el hecho de que 4 de ellos consiguieron la continencia en el primer mes. Todos refieren disfunción eréctil.

Resultados oncológicos: En el estudio patológico de la pieza se demostró en todos ellos un adenocarcinoma de próstata cuyo grado de diferenciación correspondía al de la biopsia previa. En ninguno se observó bordes positivos. Las vesículas seminales no estaban infiltradas en ningún caso. Los ganglios analizados del $7^{\circ}$ caso fueron negativos para tumor. 4 eran estadios pT2a y otros 4 pT2b. 6 pacientes han sido valorados con PSA al mes de la cirugía, siendo este menor de 0,1 en todos los casos.

\section{DISCUSIÓN}

La primera PRL fue publicada por Schuessler en 1992. A pesar de tener la colaboración de grandes laparoscopistas como Clayman y Kavoussi, la experiencia no convenció a los autores y en 1997 publicaron su pequeña casuística desaconsejando la técnica por no ofrecer ventajas sobre la cirugía convencional. En este periodo de tiempo sólo se publican dos trabajos sobre el tema: una experiencia en animales ${ }^{5}$ y un caso clínico exitoso comunicado por Raboy ${ }^{6}$. En 1998 Guillonneau y cols ${ }^{7}$ publican su experiencia preliminar en 28 casos. A partir de entonces la técnica se generalizó y varios hospitales europeos la comenzaron a realizar sistemáticamente, de tal forma que en el congreso de la AUA del 2001, se presentó un trabajo multicéntrico con 1.228 pacientes operados entre enero de 1998 y noviembre de $2000^{8}$. La técnica ha tenido pues una difusión rápida y amplia por Europa y ya existen varios hospitales americanos que han presentado sus experiencias.

Se ha comunicado una serie de variantes en la técnica descrita por Guillonneau. Vallancien coloca los trocares de diferente manera. El trocar de la línea media lo sitúa en el lado izquierdo, con lo que puede realizar la operación más cómodamente ya que se puede sentar y utilizar las pinzas y las tijeras con los trocares de la izquierda. Rassweiler ${ }^{9}$ realiza una disección ascendente de la próstata como en la cirugía abierta. Bollens ${ }^{10}$ en el Erasme Hospital de Bélgica realiza la prostatectomía por vía extraperitoneal. Con ello consigue disminuir las complicaciones intestinales, pero parece que la cirugía es más larga (tiempo medio de $330 \mathrm{~min}$ ) debido a la dificultad en la disección de las vesículas seminales. 
También se han descrito varias formas de ligar los pedículos laterales. La mayoría de los autores utiliza la coagulación bipolar. Gill en la Cleveland Clinic utiliza Endoclips. Se han utilizado EndoGias y bisturí ultrasónico, pero no hemos encontrado en la literatura que se haya utilizado el sistema de sellado de vasos LigaSure. Creemos que es un buen sistema, cómodo de utilizar, rápido y muy seguro.

Así mismo hay varias formas de realizar la anastomosis uretro-vesical. La mayoría la realiza como en la técnica descrita por Guillonneau, es decir 10 puntos sueltos con sutura reabsorbible de 3-0 y nudos intracorpóreos. Abbou ${ }^{11}$ en el Hospital Henri Mondor de Créteil realiza una sutura continua. Nosotros creemos que esta sutura no ofrece ventajas clínicas ni en el tiempo de realización. Sin embargo la utilización del sistema de sutura EndoStitch si que nos ofrece comodidad y nos ahorra bastante tiempo en esta fase quirúrgica. El único inconveniente es que encarece ligeramente la operación.

La preservación de las bandeletas neurovasculares en los casos en los que está indicado parece que puede ser una de las grandes ventajas de la PRL sobre la cirugía abierta. Al tener una mejor visión, la disección puede ser mucho más precisa. Los resultados clínicos parecen confirmar esta apreciación.

Existen varios sistemas robóticos que facilitan la operación. El más utilizado es el AESOP $3000^{\mathrm{TM}}$ (Computer Motion, Goleta, California), que es un brazo que mueve la cámara obedeciendo órdenes orales del cirujano. Otros sistemas como el Da Vinci ${ }^{\mathrm{TM}}$ (Intuitive Inc, Mountain View, California) que tienen un sistema de visión en 3D y el cirujano se coloca fuera del campo quirúrgico, no parece que aporten grandes beneficios y encarecen considerablemente el procedimiento.

Se han descrito una serie de contraindicaciones absolutas para la cirugia: insuficiencia respiratoria severa, antecedentes de aneurisma intracraneal, enfermedad cardíaca grave, cirugía pélvica importante y la oposición del paciente. Así mismo existen unas contraindicaciones relativas: cirugía abdominal inframesocólica, manipulación prostática previa (RTU, adenomectomías, biopsias múltiples, prostatitis de repetición, etc..), volúmenes prostáticos elevados (>80 gr), trata- mientos previos (hormonoterapia, radioterapia, braquiterapia) y un alto índice de masa corporal. Nosotros hemos seguido estas normas excepto en el caso 2, que se trataba de una próstata de 165 gr. La disección fue muy dificultosa y aconsejamos que por lo menos en los primeros casos no se opere pacientes con próstatas grandes. Hemos operado también pacientes con antecedentes de apendicectomías y herniorrafias y esto no nos ha causado grandes problemas ya que se pudieron liberar las adherencias fácilmente.

En la Tabla III presentamos los resultados en las series más importantes. Es difícil la comparación entre ellas, debido a las diferentes técnicas y sobre todo por la curva de aprendizaje. En todas las series existe una diferencia significativa entre las primeras operaciones y las últimas principalmente en el tiempo quirúrgico, en el porcentaje de conversiones, en el sangrado y en la tasa de complicaciones. A pequeña escala, a nosotros nos ha pasado lo mismo. En las primeras tuvimos unos tiempos quirúrgicos elevados, que fueron mejorando en los posteriores. Sin embargo nuestros datos son comparables a las otras series en porcentaje de conversiones, sangrado y tasa de complicaciones. No hemos tenido ninguna conversión a cirugía abierta, ningún paciente ha precisado transfusión y no hemos tenido complicaciones graves que precisaran reintervención. Creemos que esto es debido a que actualmente la curva de aprendizaje es menor al estar la técnica muy estandarizada. Creemos que el mayor problema es superar los pasos difíciles de la operación, como la ligadura del plexo venoso dorsal y la sutura uretro-vesical. Es aquí donde el cirujano debe de encontrar la forma de superarlos de la manera en la que el se encuentre más cómodo. La utilización de aparatos como el LigaSure o el EndoStitch puede ser muy útil en los primeros casos.

Los resultados oncológicos se exponen en la Tabla IV. Aunque los seguimientos no son largos, los controles biológicos son idénticos a los obtenidos en la cirugía abierta. Todo hace pensar que los resultados a largo plazo también serán semejantes. A destacar el hecho de que en ningún paciente de las series publicadas se ha descrito la aparición de metástasis en la zona de los puertos. 
PROSTATECTOMÍA RADICAL LAPAROSCÓPICA. RESULTADOS DE LAS PRINCIPALES SERIES

\begin{tabular}{|c|c|c|c|c|c|}
\hline & $\begin{array}{l}\text { Guillonneau } \\
\text { N: } 567\end{array}$ & $\begin{array}{l}\text { Abbou } \\
\text { N: } 134\end{array}$ & $\begin{array}{l}\text { Rassweiler } \\
\text { N: } 180\end{array}$ & $\begin{array}{l}\text { Ingolf Türk } \\
\text { N: } 125\end{array}$ & $\begin{array}{l}\text { Schulman } \\
\text { N: } \mathbf{5 0}\end{array}$ \\
\hline $\begin{array}{l}\text { Tiempo } \\
\text { Intervención (min) }\end{array}$ & 210 & 210 & $\begin{array}{c}271 \\
(150-500)\end{array}$ & $\begin{array}{c}255 \\
(135-450)\end{array}$ & 317 \\
\hline Sangrado (ml) & $\begin{array}{c}380 \\
(50-1.600)\end{array}$ & - & $\begin{array}{c}1.230 \\
(200-3.500)\end{array}$ & $\begin{array}{c}185 \\
(100-450)\end{array}$ & 680 \\
\hline Tasa transfusión & $4,9 \%$ & $3 \%$ & $31 \%$ & $2 \%$ & $13 \%$ \\
\hline Tiempo sonda (días) & $\begin{array}{c}5,8 \\
(2-30)\end{array}$ & 4,8 & $\begin{array}{c}7 \\
(5-30)\end{array}$ & $\begin{array}{c}12 \\
(5-39)\end{array}$ & 7,3 \\
\hline $\begin{array}{l}\text { Estancia } \\
\text { Hospitalaria (días) }\end{array}$ & $\begin{array}{c}6,2 \\
(2-33)\end{array}$ & 6,1 & $\begin{array}{c}10 \\
(7-27)\end{array}$ & $\begin{array}{c}8 \\
(6-14)\end{array}$ & 8,3 \\
\hline Conversión & $1,2 \%$ & $0 \%$ & $4,4 \%$ & $0 \%$ & $0,5 \%$ \\
\hline Tasa complicaciones & $17,1 \%$ & $11,2 \%$ & $18,8 \%$ & $14,4 \%$ & $16,5 \%$ \\
\hline
\end{tabular}

\section{TABLA IV}

RESULTADOS ONCOLÓGICOS DE LAS PRINCIPALES SERIES

\begin{tabular}{|c|c|c|c|c|c|}
\hline & Guillonneau & Abbou & Rassweiler & Türk & Bollens \\
\hline PTNM & $\begin{array}{l}\text { pT2: } 87,5 \% \\
\text { pT3: } 12,5 \%\end{array}$ & $\begin{array}{c}\text { pT2 } \\
75,4 \% \\
\text { pT3 } \\
24,6 \%\end{array}$ & $\begin{array}{c}\text { pT1: } 1,6 \% \\
\text { pT2: } 48,8 \% \\
\text { pT3: } 45,5 \% \\
\text { pT4: } 3,8 \%\end{array}$ & $\begin{array}{l}\text { pT2: } 61,6 \% \\
\text { pT3: } 38,4 \%\end{array}$ & $\begin{array}{c}\text { pT1: } 2,5 \% \\
\text { pT2: } 55 \% \\
\text { pT3: } 42,5 \%\end{array}$ \\
\hline $\begin{array}{l}\text { Márgenes } \\
\text { Positivos }\end{array}$ & $15 \%$ & $24,6 \%$ & $16 \%$ & $26,4 \%$ & $22 \%$ \\
\hline $\begin{array}{l}\text { PSA } \\
(<0,1 \text { control } \mathrm{ng} / \mathrm{ml})\end{array}$ & $94,7 \%$ & $89,6 \%$ & $95 \%$ & $100 \%$ & $94,3 \%$ \\
\hline
\end{tabular}

El indice de complicaciones en las diferentes series se sitúa alrededor del 15\%. Sin embargo, en ninguna se comunica mortalidad y las complicaciones graves son muy infrecuentes. Guillonneau ${ }^{12}$ en su último trabajo analiza sus complicaciones y las compara con las series más importantes de cirugía abierta y concluye que la técnica laparoscópica tiene menos morbilidad.

En nuestra serie, todas las complicaciones son de carácter leve. Las lesiones vesicales se produjeron durante la disección de las vesículas seminales. Se solucionaron en el mismo acto quirúrgico mediante sutura intracorpórea, sin dejar secuelas posteriores. Las fístulas de la anastomosis y los íleos paralíticos se solucionaron con medidas conservadoras. Únicamente la neuropraxia del primer caso le produjo una discreta discapacidad por la sensación parestésica en la extremidad inferior, pero en ningún momento presentó alteración de la deambulación. Aconsejamos, sobre todo en los primeros casos, donde la cirugía es más larga, que las zonas de apoyo del paciente sobre la cama o las perneras estén bien almohadilladas.

Parece que al ser la sutura laparoscópica más precisa que en la cirugía abierta, el tiempo de cateterización vesical puede ser más corto. Guillonneau en sus primeros pacientes lo mantuvo durante 7 días, pero ha ido reduciendo este periodo. Actualmente tiene 121 pacientes con 3 días de sondaje postoperatorio. Reducir más este periodo parece contraproducente ya que se produce con mayor frecuencia retención de orina. Nosotros de momento mantenemos la sonda por lo menos 8 días.

Los resultados con respecto a la continencia parece ser otra ventaja de la PRL frente a la prostatectomía abierta. Guillonneau obtiene un 76\% de continencia total a los 6 meses. La mitad de ellos la obtiene en el primer mes del post-opera- 
torio. Türk ${ }^{13}$ a los 9 meses tiene una tasa de continentes del $92 \%$. Varios trabajos en los que se hace una evaluación crítica en los resultados de la cirugía abierta establecen la tasa de continencia entre un 73 y un $85 \%$, a pesar de lo difícil que es valorar este dato en las diferentes series. Además parece que la continencia en la PRL se alcanza en menos tiempo después de la cirugía.

Guillonneau hace un análisis financiero entre las dos técnicas y afirma que la PRL es 1.237 dólares más barata.

Sin embargo la PRL no está exenta de críticas. Kavoussi ${ }^{14}$ en un editorial del Urology comenta los inconvenientes de esta técnica. En primer lugar dice que su duración es extremadamente larga, lo que la encarece demasiado debido a los costes del quirófano por minuto en USA. Pero reconoce que estos tiempos se reducen con la experiencia, según él a partir de la 60 PRL. Nosotros creemos que una vez que la técnica se ha desarrollado y está bien protocolizada, los tiempos quirúrgicos se reducen más rápidamente. Lo mismo ocurrió con la nefrectomía laparoscópica. Los primeros casos fueron realizados también en tiempos muy largos. Una vez sistematizada la técnica, actualmente cualquier urólogo con algo de formación laparoscópica la puede realizar en tiempos parecidos a los utilizados en la cirugía abierta.

Kavoussi se pregunta si la PRL es mejor que la cirugía abierta. Dice que en los procedimientos sobre el riñón (nefrectomía, pieloplastia...) rápidamente se demostró estadísticamente la superioridad de las técnicas laparoscópicas sobre las abiertas en lo referente a la morbilidad. Esto es verdad debido a que la incisión media infraumbilical es mejor tolerada que las lumbotomías. Pero creemos que la PRL tiene indudables ventajas sobre la abierta que con el tiempo se demostrará. De hecho la rápida difusión de la técnica en múltiples hospitales así lo hace pensar.

\section{CONCLUSIONES}

La PRL es una técnica difícil pero creemos que la curva de aprendizaje no será tan larga como en las primeras series, por lo que muchos grupos de urólogos se animarán a realizarla en un futuro no muy lejano. Los resultados oncológicos son similares a la cirugía abierta, pero ofrece pequeñas ven- tajas en muchos aspectos (sangrado, días de sondaje, estancia hospitalaria, retorno a la actividad, continencia, función sexual, cosmética, dolor postoperatorio...), lo que le hace ser una técnica muy atractiva principalmente para el paciente.

\section{REFERENCIAS}

1. TEILLAC P, ADOLFSSON J, ARAP $\mathrm{S}$, et al.: Treatment of localized disease. En "Prostate Cancer. $2^{\text {nd }}$ Internacional consultation on prostate cancer". Plymbridge Distributors Ltd. Plymouth. 1999; 256-280.

2. SCHLUESSER WW, KAVOUSSI LR, CLAYMAN RV, et al.: Laparoscopic radical prostatectomy: initial case report (abstract). J Urol suppl 1992; 147: 246.

3. SCHLUESSER WW, SCHULAM PG, CLAYMAN RV, et al.: Laparoscopic radical prostatectomy: initial short-term experience. Urology 1997; 50: 854-857.

4. GUILLONNEAU B, VALLANCIEN G.: Laparoscopic radical prostatectomy: the Montsouris technique. $J$ Urol 2000; 163: 1643-1649.

5. PRICE DT, CHARI RS, NEIGHBORS JD, et al.: Laparoscopic radical prostatectomy in the canine model. J Laparoscopic Surgery 1996; 6: 405-412.

6. RABOY A, FERZLI G, ALBERT P.: Initial experience with extraperitoneal endoscopic radical retropubic prostatectomy. Urology 1997; 50: 849-853.

7. GUILLONNEAU B, CATHELINEAU X, BARRET E, et al.: Prostatectomie radicale coelioscopique. Premiére evaluation aprés 28 interventions. Presse Med 1998; 27: 1570-1575.

8. SULSER T, GUILLONNEAU B, VALLANCIEN G et al.: Complications and initial experience with 1228 laparoscopic radical prostatectomies at 6 european centers (abstract). J Urol suppl 2001; 165: 150.

9. RASSWEILER J, SENTKER L, SEEMANN O, et al.: Laparoscopic radical prostatectomy with the Heilbronn technique: an analysis of the first 180 cases. J Urol 2001; 166: 2101-2108.

10. BOLLENS R, VANDEN BOSSCHE M, ROUMEGUE$\mathrm{RE}$ Th et al.: Extraperitoneal Laparoscopic radical prostatectomy. Eur Urol 2001; 40: 65-69.

11. HOZNEK A, SALOMON L, OLSSON LE, et al.: Laparoscopic radical prostatectomy. The Créteil experience. Eur Urol 2001; 40: 38-45.

12. GUILLONNEAU B, ROZET F, CATHELINEAU X, et al.: Perioperative complications of Laparoscopic radical prostatectomy: the Montsouris 3-year experience. J Urol 2002; 167: 51-56.

13. TÜRK I, DEGER S, WINKELMANN B, SCHÖNBERGER B, LOENING SA: Laparoscopic radical prostatectomy. Eur Urol 2001; 40: 46-53.

14. KAVOUSSI LR: Laparoscopic radical prostatectomy: irrational exuberance?. Urology 2001; 58: 503-505.

Dr. J. Estébanez Zarranz

C/ Mariano de Los Cobos, 89

47014-Valladolid

(Trabajo recibido el 10 de agosto 2002) 